\title{
Enhancing Heat and Mass Transfer in Adsorption Heat Pumps Using Advanced Metal Organic Framework Materials
}

\author{
Dr. Raya AL-Dadah \\ School of Engineering \\ University of Birmingham \\ Birmingham, United Kingdom \\ r.k.al-dadah@bham.ac.uk
}

\section{Abstract}

The ever-increasing global energy consumption and the heavy reliance on fossil fuels posing significant worldwide economic and environmental challenges, particularly the increased greenhouse gas (GHG) emissions and the global warming. Current technologies for heating (central heating gas boilers), cooling (electrically driven vapour compression refrigeration systems) and water desalination (Reverse Osmosis) are energy intensive and consume significant amounts of fossil fuels and contribute to GHG emissions. There is a huge amount of unexploited energy including overnight cheap electricity, intermittent renewables and industrial waste heat which can be exploited to reduce fossil fuel consumption and CO2 emission. Heat powered adsorption technology offer potential of using various sources of low-grade thermal energy for cooling, heating and water desalination. However, commercially available adsorption systems utilise silica gel or zeolite adsorbent materials which have limited water adsorption capabilities thus they suffer from being bulky, expensive and have low efficiency.

This keynote speech describes the author's research work for enhancing the performance of heat powered adsorption systems using advanced microporous adsorbent materials known as Metal Organic Framework materials (MOF) and various techniques for enhancing heat and mass transfer in adsorber beds. Metal Organic Frameworks (MOFs) are meso-porous materials with exceptionally high porosity, large surface area (up to $5500 \mathrm{~m} 2 / \mathrm{g}$ ) and superior water uptake capacity (up to $1.5 \mathrm{kgw} / \mathrm{kgads})$. MOFs have two main components: the organic linkers which act as struts that bridge metal centres known as inorganic building units. The two main components are connected to each other by coordination bonds to form a network with defined topology. As MOF structures can be constructed with a diversity of metal clusters/ions and organic components to generate porous materials with high surface area, they possess more advantages to tune their properties via structure design. Moreover, the modification of MOFs via functionalisation or post-synthetic modification could offer routes to enhance their thermophysical properties.

At University of Birmingham, the author's research work included the synthesis and characterisation of several MOF materials (MIL101-(Cr), MIL100(Fe), Aluminium Fumarate, CPO27-(NI)) in terms of water adsorption isotherms and kinetics, thermophysical properties like thermal conductivity, specific heat capacity, density and pores surface area. The research work also included enhancing the thermal conductivity by addition of highly conductive graphene oxide and enhancing the adsorption isotherm by adding hygroscopic calcium chloride into the synthesis process. Results showed that adding 5\% GrO to MIL101(Cr) increased thermal conductivity by 2.5 times while suspending MIL101(Cr) in $\mathrm{CaCl} 2 \mathrm{salt}$ solution with $8 \%$ concentration resulted in modifying the shape of the water adsorption isotherm increasing MIL101(Cr) at low relative pressure value of 0.2 reaching 5 times that of neat MIL101(Cr). Finally, research work at UoB included various methods of integrating the MOF material in the adsorber beds namely packing and coating and the use of various types of heat exchangers like rectangular finned tubes and wire finned tubes in the adsorber beds. Results showed that aluminium fumarate MOF coated wire finned tube heat exchangers enhanced the performance of adsorption system significantly where specific daily water production reached $15 \mathrm{~m} 3 /$ tonne/day and the specific cooling power reached $550 \mathrm{~kW} /$ tonne. 